\title{
Reflorestamento e Recuperação de Atributos Químicos e Físicos do Solo
}

\author{
José Henrique Tertulino Rocha ${ }^{1 \star}$, Alessandro José Marques Santos², \\ Fernanda Asti Diogo ${ }^{3}$, Clarice Backes², Augusto Gabriel Claro de Melo , \\ Karla Borelli ${ }^{1}$, Tiago de Oliveira Godinho ${ }^{5}$ \\ ${ }^{1}$ Departamento de Ciências Florestais, Escola Superior de Agricultura Luiz de Queiroz - ESALQ, \\ Universidade de São Paulo - USP, Piracicaba/SP, Brasil \\ ${ }^{2}$ Curso de Zootecnia, Universidade Estadual de Goiás - UEG, São Luís de Montes Belos/GO, Brasil \\ ${ }^{3}$ Departamento de Ciência Florestal, Universidade Estadual Paulista - UNESP, Botucatu/SP, Brasil \\ ${ }^{4}$ Curso de Engenharia Florestal, Faculdade de Ensino Superior e Formação Integral - FAEF, Garça/SP, Brasil \\ ${ }^{5}$ Instituto Capixaba de Pesquisa, Assistência Técnica e Extensão Rural - INCAPER, Domingos Martins/ES, Brasil
}

\begin{abstract}
RESUMO
Objetivou-se com o trabalho avaliar a influência do reflorestamento com essências nativas da Floresta Estacional Semidecidual, plantio de eucalipto e pastagem nos atributos químicos e físicos do solo de áreas degradadas. Foram selecionadas cinco áreas na bacia hidrográfica do Rio Aguapeí, na cidade de Garça, SP, sendo dois reflorestamentos com essências nativas, com 4 e 9 anos, um plantio de eucalipto, uma área de pastagem e um fragmento florestal utilizado como referência. $\mathrm{O}$ fragmento florestal apresentou a menor soma de bases e os maiores teores de $\mathrm{P}, \mathrm{C}_{\text {org }}$ e CTC. Ele também apresentou a menor resistência à penetração, maior diâmetro e estabilidade dos agregados. Os atributos químicos e físicos do solo das áreas reflorestadas diferiram dos observados no fragmento florestal, mostrando que o período de recuperação não foi suficiente para restaurar esses atributos. Porém o reflorestamento com 9 anos melhorou significativamente nesses atributos do solo, tendendo a aproximar-se aos do fragmento florestal.
\end{abstract}

Palavras-chave: indicadores ecológicos, qualidade do solo, restauração florestal.

\section{Reforestation and Recovery of Soil Chemical and Physical Attributes}

\begin{abstract}
The objective with this study was to evaluate the influence of reforestation with native species from seasonal semideciduous forest, eucalyptus plantation, and pasture in the soil chemical and physical properties of degraded areas. Five sites were selected in the watershed of the Aguapeí River in the Garça city, Sao Paulo state: two reforestation areas with native species, with four and nine years old; a reforestation area with eucalyptus plantation, a pasture area, and a forest fragment used as control. The forest fragment presented the lowest exangeable bases and the highest levels of P, SOC and CEC; it also showed the lowest penetration resistance, and the largest diameter and aggregate stability. The chemical and physical soil attributes of the reforestation areas differed from those of the forest fragment (control), showing that the recovery period was not sufficient to restore these attributes. However, the 9-year-old reforestation area presented significant improvement of these soil attributes, tending to approach those of the control area.
\end{abstract}

Keywords: ecological indicators, soil quality, forest restoration. 


\section{INTRODUÇÃO}

As conversões de áreas naturais em ambientes agrícolas implicam em grandes mudanças na estrutura e no funcionamento dos ecossistemas. Devido a esse fato, diversos estudos foram realizados para dimensionar a grandeza dessas mudanças (Cerri et al., 1985; Trumbore et al., 1995; Moreira \& Malavolta, 2004) ou até mesmo prevê-las (Silveira et al., 2000). Porém, trabalhos que objetivam avaliar como ocorre a recuperação desses ecossistemas quando são reflorestados são menos comuns.

O monitoramento da qualidade ambiental é fundamental para avaliar se os esforços efetuados nos programas de restauração florestal são viáveis. Para efetuar esse monitoramento, diversos fatores devem ser considerados, dentre eles os parâmetros biométricos do componente arbóreo (Melo et al., 2007) e a qualidade do solo (D’Andréa et al., 2002) se destacam.

A qualidade do solo pode ser conceituada como a capacidade de sustentar a produtividade biológica, manter ou melhorar a qualidade ambiental e contribuir para a saúde das plantas, dos animais e humana (Brady \& Weil, 2002). O nível dessa qualidade pode ser medido por meio da quantificação dos atributos químicos, físicos e/ou biológicos (Melloni et al., 2008; Moreira \& Malavolta, 2004). Esses atributos devem ser comparados com os de solos sob condições naturais ou áreas pouco perturbadas para inferir o grau de degradação em que essas áreas se encontram (Islam \& Weil, 2000).

De modo geral, sistemas de cultivo que proporcionam menor revolvimento do solo e aporte frequente de serapilheira tendem a melhorar as propriedades físicas (Silva et al., 2005; Araújo et al., 2007; Melloni et al., 2008) e químicas do solo (Bayer \& Mielniczuk, 1997; Souza \& Alves, 2003; Oliveira et al., 2004). Um solo que apresenta boas características físicas segundo Reichert et al. (2003) é aquele capaz de: i) permitir a infiltração, retenção e disponibilidade de água às plantas, córregos e subsuperfícies; ii) responder ao manejo e resistir à degradação; iii) permitir as trocas de calor e de gases com a atmosfera e raízes de plantas; e iv) possibilitar o crescimento das raízes. $\mathrm{E}$ as boas características químicas do solo, segundo Meurer (2007), são aquelas capaz de: i) fornecer os nutrientes para o desenvolvimento das plantas; ii) não apresentar elementos tóxicos e metais pesados em concentrações nocivas; iii) apresentar capacidade de promover reações de sorção; e iv) não apresentar nível nocivo de salinidade.

Objetivou-se com o trabalho avaliar a influência do reflorestamento com essências nativas da Floresta Estacional Semidecidual, plantio de eucalipto e pastagem nos atributos químicos e físicos do solo de áreas degradadas.

\section{MATERIAL E MÉTODOS}

O trabalho foi realizado nas microbacias do Ribeirão da Garça e Ribeirão Barreiro, pertencentes à bacia hidrográfica do Rio Aguapeí (UGRHI 20), na

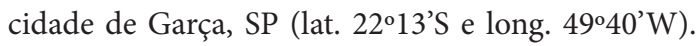
O clima da região é o Cfa, segundo a classificação de Köppen (Alvares et al., 2014). A precipitação média anual é de $1.320 \mathrm{~mm}$, temperatura média de $20,7^{\circ} \mathrm{C}$ e altitude de $600 \mathrm{~m}$. A vegetação original da área é a Floresta Estacional Semidecidual.

Para realização deste estudo foram selecionadas cinco áreas: dois reflorestamentos com essências nativas para fins de restauração, o primeiro com 4 anos (REF1) e o segundo com 9 anos (REF2) de implantação; um reflorestamento de um híbrido de Eucalyptus urophylla com Eucalyptus grandis (EUCA) com 3 anos; uma pastagem extensiva com Brachiaria decumbens (PAS) e um fragmento florestal pouco perturbado (FFPP).

Essas áreas foram desmatadas por volta dos anos de 1910 e 1920, cultivadas com café por um longo período, sendo posteriormente substituída por pastagem. Durante esse período, o solo dessas áreas foi bastante alterado. Antes do reflorestamento, essas áreas foram ocupadas por mais de 20 anos com pastagem extensiva de Brachiaria decumbens com baixos investimentos em tratos culturais. Os reflorestamentos, tanto das essências nativas como o de eucalipto, foram instalados sob o sistema de cultivo mínimo, no espaçamento de $3 \times 2 \mathrm{~m}$. Em ambos foram aplicados aproximadamente $100 \mathrm{~kg} \mathrm{ha}^{-1} \mathrm{de} \mathrm{N}$, $80 \mathrm{~kg} \mathrm{ha}^{-1} \mathrm{de}_{2} \mathrm{O}_{5}$ e $100 \mathrm{~kg} \mathrm{ha}^{-1}$ de $\mathrm{K}_{2} \mathrm{O}$ divididos em adubação de base e cobertura.

$\mathrm{O}$ fragmento florestal consiste em um fragmento da Floresta Estacional Semidecidual que nunca sofreu 
corte raso, entretanto existem indícios da extração seletiva de madeira em seu interior. Essa área vem sendo protegida da intervenção humana desde o início da década de 1960, estando em um estágio avançado de regeneração.

As áreas estão localizadas em um raio de $10 \mathrm{~km}$, com declividade média variando de $5 \%$ a $7 \%$, situadas no terço inferior da topossequência. O solo das áreas é o Argissolo Vermelho-Amarelo abrupto (Embrapa, 2006). A análise de textura mostra que as áreas apresentam o horizonte $\mathrm{A}$ arenoso, com teor médio de argila de $73 \mathrm{~g} \mathrm{~kg}^{-1}$ (Tabela 1).

Em cada área foram lançadas ao acaso quatro parcelas de $20 \times 20 \mathrm{~m}\left(400 \mathrm{~m}^{2}\right)$, onde foram coletadas em um transecto diagonal as amostras de solo para as análises de fertilidade, porcentagem de agregados, densidade do solo e porosidade total. Nessas áreas foi medida também a resistência à penetração.

A coleta de solo para análise química foi efetuada com uma sonda, sendo coletadas 10 amostras simples de solo por parcela, que foram misturadas, formando uma amostra composta. Essas foram analisadas para determinação do $\mathrm{pH}$, carbono orgânico $\left(\mathrm{C}_{\mathrm{org}}\right)$, CTC, saturação por bases, além dos teores de $\mathrm{P}, \mathrm{K}, \mathrm{Ca}, \mathrm{Mg}$ e $\mathrm{H}+\mathrm{Al}$, seguindo a metodologia proposta por Van Raij et al. (2001). O teor de $\mathrm{C}_{\text {org }}$ foi determinado pelo método de Walkley \& Black (1934) e o estoque de carbono foi determinado pelo produto do teor de carbono na camada de $0-20 \mathrm{~cm}$ com a densidade da

Tabela 1. Textura da camada de $0-20 \mathrm{~cm}$ do em reflorestamentos com espécies nativas com quatro (REF1) e nove (REF2) anos, reflorestamento com Eucalyptus urophylla $\mathrm{x}$ Eucalyptus grandis (EUCA), pastagem (PAS) e fragmento florestal pouco perturbado (FFPP), Garça, SP.

Table 1. Texture of the $0-20 \mathrm{~cm}$ soil layer in reforestation with native species with four (REF1) and nine (REF2) years old, reforestation with Eucalyptus urophylla $\mathrm{x}$ Eucalyptus grandis (EUCA), area of pasture (PAS) and little disturbed forest fragment (FFPP), Garça, SP.

\begin{tabular}{cccc} 
& Areia & Silte & Argila \\
\cline { 2 - 4 } Área & & $\ldots \ldots \ldots \ldots \ldots . . . \mathbf{g ~ k g}^{-1 \ldots \ldots \ldots \ldots \ldots . . .}$ & \\
REF1 & 870 & 61 & 69 \\
REF2 & 840 & 65 & 95 \\
EUCA & 866 & 59 & 75 \\
PAS & 896 & 52 & 52 \\
FFPP & 866 & 61 & 73 \\
\hline
\end{tabular}

camada. A densidade do solo foi obtida por meio da massa seca (em estufa a $105^{\circ} \mathrm{C}$ por $24 \mathrm{~h}$ ) das amostras de solo indeformadas coletadas com auxílio do anel volumétrico dividida por seu volume (Blake \& Hartge, 1986)

A determinação da percentagem de agregados por classe de tamanho pelo método seco, foi realizada conforme a metodologia descrita pela Embrapa (1997). Para essas análises foram utilizadas $100 \mathrm{~g}$ das amostras compostas, que foram secas ao ar por 72 horas e posteriormente passadas nas peneiras de $2 ; 1 ; 0,5$; 0,$25 ; 0,125 \mathrm{~mm}$ e um fundo. Cada amostra ficou por um período de 15 minutos no agitador de peneiras. Posteriormente, foi determinada a massa de solo que ficou em cada peneira.

O diâmetro médio ponderado (DMP), diâmetro médio geométrico (DMG) e o índice de estabilidade de agregados (IEA\%) foram calculados segundo as Equações 1-3, propostas por Castro et al. (1998):

$\mathrm{DMP}=\left[\Sigma\left(\mathrm{MA}_{\mathrm{i}} \mathrm{d}_{\mathrm{i}} / \mathrm{MAT}\right)\right]$

$\mathrm{DMG}=\mathrm{EXP} \sum_{\mathrm{I}=1}^{\mathrm{N}} \frac{\text { MAi.logdi }}{\mathrm{MAT}}$

IEA $\%=($ MAT - FRAÇÃO $<0,25 \mathrm{~mm}) \times 100$

em que: $\mathrm{MA}_{\mathrm{i}}$ é a massa dos agregados dentro de cada classe; $d_{i}$ é o diâmetro médio da classe; e MAT é a massa total dos agregados.

A resistência mecânica do solo à penetração foi mensurada utilizando-se penetrômetro eletrônico modelo PLG 1020, Falker. Dentro de cada parcela foram efetuadas quatro medidas, na profundidade de 0 a $30 \mathrm{~cm}$. Os dados obtidos foram subdivididos em seis faixas de profundidade ( $5 \mathrm{~cm}$ de espessura), sendo considerado o valor médio em cada faixa.

Os dados foram submetidos ao teste de normalidade (Shapiro-Wilk) e homocedasticidade (Box-Cox) e, quando necessário, os dados foram corrigidos. Os resultados foram submetidos à análise de variância por meio do teste $\mathrm{F}$ e comparação de médias por meio do teste LSD. A relação entre a CTC e o pH, acidez potencial e $\mathrm{C}_{\text {org }}$ do solo foi avaliada por meio de análise de regressão e a relação entre o teor de $\mathrm{C}_{\text {org }}$ do solo com os atributos de agregação foi avaliada pelo coeficiente de correlação de Pearson. Foi utilizado o software SAS 9.1 for Windows (SAS, 2008). 


\section{RESULTADOS E DISCUSSÃO}

\subsection{Atributos químicos}

Os atributos químicos do solo sob os reflorestamentos (REF1, REF2 e EUCA) apresentaram poucas variações quando comparados ao da pastagem (PAS). Porém, grandes diferenças foram observadas quando comparados ao do fragmento florestal pouco perturbado (FFPP) (Tabela 2).

$\mathrm{O}$ menor valor de $\mathrm{pH}$ e o maior valor de $\mathrm{H}+\mathrm{Al}$ foram observados no FFPP, valores esses que diferiram $(\mathrm{p}<0,0001)$ das demais áreas. O FFPP também foi a área que apresentou os menores teores de potássio $(\mathrm{K})$, cálcio $(\mathrm{Ca})$, magnésio $(\mathrm{Mg})$ e, consequentemente, os menores valores de soma de bases (SB) e saturação por bases (V\%). Os maiores valores desses atributos em REF1, REF2, EUCA e PAS é resultado das correções e fertilizações feitas nessas áreas em seu longo período de cultivo (de 1920 até o momento do reflorestamento). Valores similares de Ca, Mg e SB foram encontrados por Rodrigues et al. (2007) em um Argissolo distrófico sob Floresta Estacional Semidecidual no estado de Minas Gerais e por Godinho et al. (2013) em um Latossolo Vermelho-Amarelo sob Floresta Estacional Semidecidual no Estado do Espírito Santo. O fato de solos com baixa disponibilidade de nutrientes serem capazes de manter uma vegetação arbórea se deve a alta eficiência no uso dos nutrientes, devido ao processo de ciclagem (Vital et al., 2004).

As áreas que apresentaram a maior disponibilidade de P no solo foram o FFPP e o REF2, diferindo ( $p<0,0001)$ das demais (Tabela 2). A maior disponibilidade de $\mathrm{P}$ foi observada nas áreas que apresentavam os maiores teores de $\mathrm{C}_{\text {org }}$ no solo (Figura 1a). Isso se deve ao fato de que algumas frações da matéria orgânica do solo, principalmente os ácidos fúlvicos, ocupam os sítios de absorção do P no solo, aumentando sua disponibilidade (Brady \& Weil, 2002).

O FFPP foi a área que apresentou maior CTC, diferindo estatisticamente $(\mathrm{p}<0,0001)$ das demais áreas, mesmo com o menor valor de $\mathrm{pH}$. Esse fato é melhor compreendido analisando-se o teor de carbono orgânico das áreas (Figura 1a). Mesmo tendo suas cargas de caráter variável, devido seu baixo ponto de carga zero $(\mathrm{PCZ}<3,0)$, em baixo valor de $\mathrm{pH}$, a matéria orgânica do solo ainda apresenta cargas negativas (Alleoni et al., 2009). Em solos de regiões de clima tropical a MOS, mesmo que em pequenas quantidades, contribui com mais de 50\% da CTC devido à reduzida atividade das argilas (Prezotti, 2007). O teor de MOS, assim como o carbono orgânico do solo (COS), tem sido utilizado como indicador de alterações do solo, sendo menor em condições estressantes. O REF2 apresentou leve recuperação dessas características em relação à área de pastagem. Valores similares de CTC foram encontrados por Leite (2001) em áreas de pastagem e floresta nativa.

Como a CTC dos solos altamente intemperizados é dependente dos atributos da acidez ( $\mathrm{pH}$ e $\mathrm{H}+\mathrm{Al}$ ) e da matéria orgânica (representado na equação pelo carbono orgânico - $\mathrm{C}_{\text {org }}$ ), seu comportamento pode ser explicado $(\mathrm{p}<0,001)$ por meio da Equação 4, sendo ambas variáveis significativas $\left({ }^{* *} \mathrm{p}<0,01\right)$.

$\mathrm{CTC}=-109,604+21,959 \mathrm{pH}^{* *}+3,935 \mathrm{C}_{\text {org }}{ }^{* *}+$ $1,159(\mathrm{H}+\mathrm{Al})^{* *} \mathrm{R} 2=0,99$

Tabela 2. Atributos químicos da camada de $0-20 \mathrm{~cm}$ do solo em reflorestamentos com espécies nativas com quatro (REF1) e nove (REF2) anos, reflorestamento com Eucalyptus urophylla x Eucalyptus grandis (EUCA), pastagem (PAS) e fragmento florestal pouco perturbado (FFPP), Garça, SP.

Table 2. Chemical attributes of soil in the layer of 0-20 cm depth in reforestation with native species with four (REF1) and nine (REF2) years old, reforestation with Eucalyptus urophylla x Eucalyptus grandis (EUCA), area of pasture (PAS) and little disturbed forest fragment (FFPP), Garça, SP.

\begin{tabular}{|c|c|c|c|c|c|c|c|c|c|}
\hline \multirow{2}{*}{ Área } & \multirow{2}{*}{$\underset{\mathrm{CaCl}_{2}}{\mathrm{pH}}$} & \multirow{2}{*}{$\begin{array}{c}P_{\text {resina }} \\
\mathbf{m g ~ d m}^{-3}\end{array}$} & $\mathrm{H}+\mathrm{Al}$ & $\mathbf{K}$ & $\mathrm{Ca}$ & $\mathrm{Mg}$ & SB & CTC & \multirow{2}{*}{ V\% } \\
\hline & & & \multicolumn{6}{|c|}{$\mathrm{mmol} \mathrm{dm}^{-3}$} & \\
\hline REF1 & $4,9 \mathrm{a}^{*}$ & $2,8 \mathrm{~d}$ & $16,2 \mathrm{~b}$ & $1,12 \mathrm{~b}$ & $8,9 \mathrm{~b}$ & $4,3 \mathrm{a}$ & $14,3 \mathrm{~b}$ & $30,5 \mathrm{bc}$ & $47,0 \mathrm{c}$ \\
\hline REF2 & $4,9 \mathrm{a}$ & $7,7 \mathrm{a}$ & $19,4 \mathrm{~b}$ & $1,43 \mathrm{a}$ & $15,1 \mathrm{a}$ & $5,3 \mathrm{a}$ & $21,9 \mathrm{a}$ & $41,3 \mathrm{~b}$ & $53,0 \mathrm{~b}$ \\
\hline EUCA & $5,3 \mathrm{a}$ & $4,4 \mathrm{c}$ & $13,7 \mathrm{~b}$ & $1,05 \mathrm{~b}$ & $13,7 \mathrm{a}$ & $6,0 \mathrm{a}$ & $20,7 \mathrm{a}$ & $34,4 \mathrm{bc}$ & $60,3 a$ \\
\hline PAS & $5,0 \mathrm{a}$ & $2,6 \mathrm{~d}$ & $13,6 \mathrm{~b}$ & $1,44 \mathrm{a}$ & $6,4 \mathrm{~b}$ & $4,4 \mathrm{a}$ & $12,3 b$ & $25,9 c$ & $47,3 \mathrm{c}$ \\
\hline FFPP & $3,4 \mathrm{~b}$ & $5,7 \mathrm{~b}$ & $81,1 \mathrm{a}$ & $0,88 \mathrm{c}$ & $2,2 \mathrm{c}$ & $1,3 \mathrm{~b}$ & $4,4 \mathrm{c}$ & $85,5 \mathrm{a}$ & $5,3 \mathrm{~d}$ \\
\hline CV\% & 1,42 & 8,24 & 21,39 & 8,82 & 4,93 & 26,27 & 6,79 & 14,93 & 4,57 \\
\hline
\end{tabular}

${ }^{*}$ Letras distintas na coluna indicam diferença a nível de $5 \%$ de probabilidade pelo teste LSD 
O maior estoque de carbono orgânico do solo foi observado no fragmento florestal pouco perturbado (Figura 1b), que diferiu $(\mathrm{p}<0,001)$ dos demais. Os dois reflorestamentos com essências nativas apresentaram estoque de carbono superior ao da pastagem e ao do reflorestamento com eucalipto. Fato justificado pela maior deposição de resíduos quando comparada à da área de pastagem, evidenciado por Brun (2008). A maior qualidade e diversidade dos resíduos orgânicos gerados por florestas nativas aumentam a atividade biológica do solo e com isso elevam a velocidade de transformação desses resíduos em frações húmicas da matéria orgânica do solo (Lima et al., 2008).

Considerando que o estoque de carbono da pastagem antecessora ao reflorestamento de 9 anos fosse o mesmo da área de pastagem avaliada nesse estudo, observa-se que em nove anos ocorreu um ganho de $4,04 \mathrm{Mg} \mathrm{ha}^{-1}$ de carbono na camada de $0-20 \mathrm{~cm}$, ou seja, um ganho de $0,45 \mathrm{Mg} \mathrm{ha}^{-1} \mathrm{ano}^{-1}$. Se esse acréscimo de carbono for constante, serão necessários mais 12 anos para que esse reflorestamento atinja o estoque de carbono observado no fragmento florestal pouco perturbado.

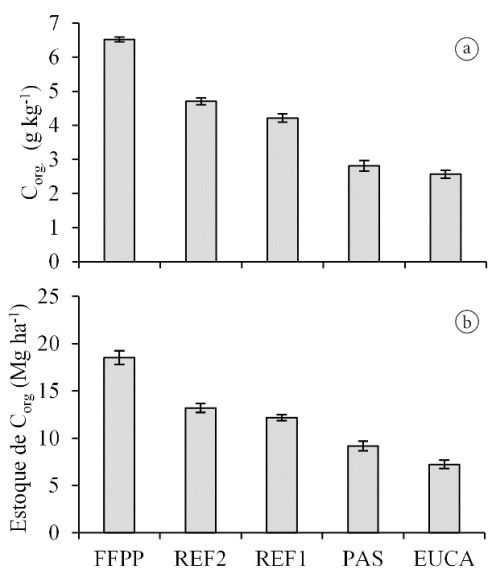

Figura 1. Teor de carbono orgânico no solo (a) e estoque de carbono orgânico na camada de $0-20 \mathrm{~cm}$ de profundidade (b) do reflorestamento com essências nativas com 4 (REF1) e com 9 (REF2) anos, do reflorestamento com Eucalyptus urophylla $\mathrm{x}$ Eucalyptus grandis (EUCA), da área de pastagem (PAS) e do fragmento florestal pouco perturbado (FFPP); as barras indicam o erro padrão da média $(\mathrm{n}=4)$.

Figure 1. Content of soil organic carbon (a) and soil organic carbon amount in the layer $0-20 \mathrm{~cm}$ depth (b) in reforestation with native species with four (REF1) and nine (REF2) years old, reforestation with Eucalyptus urophylla $\mathrm{x}$ Eucalyptus grandis (EUCA), area of pasture (PAS) and little disturbed forest fragment (FFPP). The bars indicate the standard error of the mean $(n=4)$.

\subsection{Atributos físicos}

$\mathrm{Na}$ área de pastagem foi observada maior densidade na camada de $0-20 \mathrm{~cm}$ do solo $\left(1,68 \mathrm{Mg} \mathrm{m}^{-3}\right)$, diferindo $(\mathrm{p}=0,002)$ das demais áreas, que apresentaram densidade média de $1,42 \mathrm{Mg} \mathrm{m}^{-3}$. Tal fato provavelmente é devido ao pisoteio animal.

$\mathrm{A}$ área que apresentou o maior conteúdo de $\mathrm{C}_{\text {org }}$ (FFPP) também foi a área que apresentou o maior diâmetro médio geométrico (DMG) (Figura 2a), maior diâmetro médio ponderado dos agregados (DMP) (Figura 2b) e maior índice de estabilidade dos agregados (IEA) (Figura 2c). As áreas que apresentaram menor DMP, DMG e IEA foram as áreas de pastagem
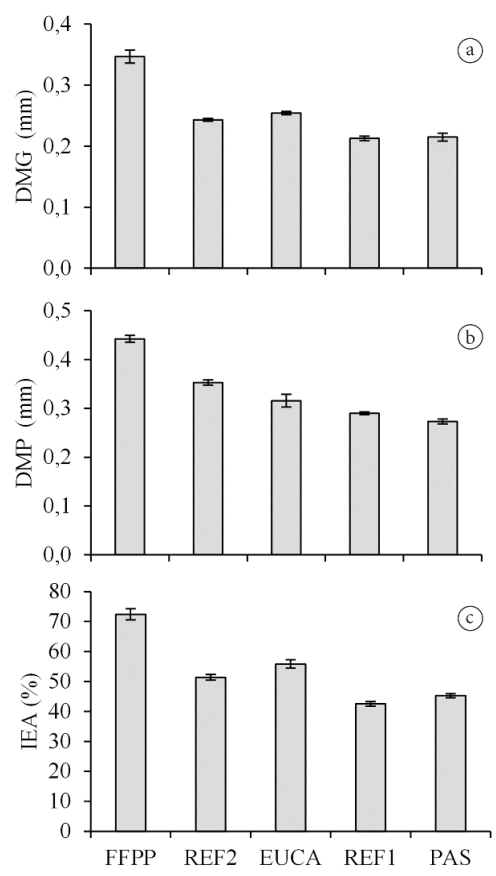

Figura 2. Diâmetro médio geométrico (DMG), diâmetro médio ponderado dos agregados (DMP) e índice de estabilidade de agregados (IEA) do reflorestamento com essências nativas com 4 (REF1) e com 9 (REF2) anos, do reflorestamento com Eucalyptus urophylla $\mathrm{x}$ Eucalyptus grandis (EUCA), da área de pastagem (PAS) e do fragmento florestal pouco perturbado (FFPP); barras indicam o erro padrão da média $(\mathrm{n}=4)$.

Figure 2. Geometric mean diameter (DMG), weighted average diameter of aggregates (DMP) and aggregate stability index (IEA) in reforestation with native species with four (REF1) and nine (REF2) years old, reforestation with Eucalyptus urophylla $\mathrm{x}$ Eucalyptus grandis (EUCA), area of pasture (PAS) and little disturbed forest fragment (FFPP). Bars indicate the standard error of the mean $(n=4)$. 


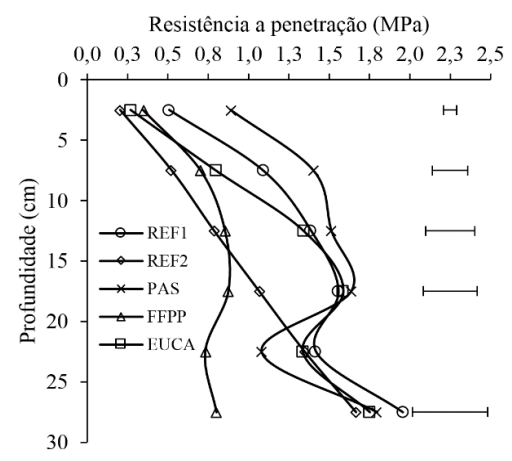

Figura 3. Resistência à penetração em reflorestamento com essências nativas com 4 (REF1) e com 9 (REF2) anos, do reflorestamento com Eucalyptus urophylla $\mathrm{x}$ Eucalyptus grandis (EUCA), da área de pastagem (PAS) e do fragmento florestal pouco perturbado (FFPP); barras indicam a diferença mínima significativa.

Figure 3. Penetration resistance in reforestation with native with four (REF1) and nine (REF2) years of age, reforestation with Eucalyptus urophylla x Eucalyptus grandis (EUCA), area of pasture (PAS) and little disturbed forest fragment (FFPP). Bars indicate the least significant difference.

e reflorestamento de 4 anos (Figuras 2a, 2b e 2c). O teor de $\mathrm{C}_{\text {org }}$ do solo apresentou correlação com o $\operatorname{DMP}(\mathrm{r}=0,83-\mathrm{p}<0,001)$, com o DMG $(\mathrm{r}=0,72-$ $p<0,001)$ e com o IEA $(r=0,64-p=0,002)$. Esse fato corrobora a relação estreita entre a matéria orgânica do solo e sua agregação (Brady \& Weil, 2002). Castro et al. (1998) observaram altos coeficientes de correlação entre esses atributos de agregação e o teor de carbono orgânico do solo ao avaliarem sistemas de plantio direto e convencional em diferentes sucessões de culturas.

Conforme explicado por Kiehl (1979), quanto maior a intensidade do manejo, maior o rompimento dos agregados do solo, com isso, menor serão o DMG, o DMP e o IEA. As áreas avaliadas recebem baixa intensidade de manejo ou não são manejadas. A baixa agregação observada para os reflorestamentos (REF1, REF2 e EUCA) e para a pastagem é reflexo de um manejo intensivo sofrido no passado, necessitando de um longo tempo de recuperação. Essas variáveis podem indicar que esse solo possui baixa capacidade de infiltração e armazenamento de água, podendo ser facilmente erodido. Segundo Kiehl (1979), os solos com DMG inferior a $0,5 \mathrm{~mm}$ são solos pouco agregados. Todos os solos analisados, até mesmo em FFPP, apresentaram DMP inferior a $0,5 \mathrm{~mm}$, isso ocorreu devido à textura arenosa desses solos (Tabela 1).
Vale ressaltar que solos com essa textura apresentam menor capacidade de agregação.

Os resultados das variáveis de agregação analisadas (DMG, DMP e IEA) foram inferiores aos observados por Castro et al. (1998), porém esses autores trabalharam com um Latossolo Roxo, solo que apresenta teor de argila maior que o do solo estudado. Além de outros fatores, a argila tem um importante papel na agregação e na estabilidade dos agregados.

Os menores valores de resistência à penetração em todas as camadas avaliadas foram observados no FFPP, valores esses que não se diferenciaram $(\mathrm{p}<0,001)$ dos do reflorestamento de 9 anos e da área de eucalipto nas camadas até $10 \mathrm{~cm}$ de profundidade e dos do reflorestamento de 9 anos até $20 \mathrm{~cm}$ de profundidade, diferenciando-se nas demais profundidades. A área que apresentou maior resistência à penetração foi a PAS, diferindo $(\mathrm{p}<0,001)$ das demais áreas até a camada de $10 \mathrm{~cm}$, devido ao intenso pisoteio animal. Nas camadas inferiores, a resistência à penetração da área de pastagem diferiu apenas do FFPP (Figura 3).

O comportamento observado na Figura 3, em que ocorre aumento da resistência à penetração até a camada de $20 \mathrm{~cm}$, com redução, posteriormente, na camada de $25 \mathrm{~cm}$ e um novo aumento nas camadas inferiores, é característico dos Argissolos, nos quais na camada próxima a $25 \mathrm{~cm}$ de profundidade se encontra o horizonte eluvial, que é bastante friável, e logo abaixo ocorre uma mudança abrupta de textura, aumentando a resistência à penetração. Comportamentos similares foram observados por Abreu et al. (2004) e Reinert et al. (2008). Valores similares de resistência à penetração para o FFPP foram observados por Beutler et al. (2001) em áreas de cerrado.

\section{CONCLUSÕES}

Os atributos químicos e físicos do solo das áreas reflorestadas e sob pastagem diferiram dos do fragmento florestal, mostrando que o período de recuperação não foi suficiente para reestabelecer esses atributos. Porém, o reflorestamento de 9 anos melhorou significativamente esses atributos do solo, tendendo a aproximá-los aos do fragmento florestal.

Como demonstrado no presente trabalho, é inquestionável o fato de que os atributos do solo, em florestas nativas, são um componente e indicador a ser 
levado em conta em estudos de diversidade e produtividade da floresta. Tais estudos devem ser considerados nos planos de manejo florestal e de restauração de florestas nativas degradadas, principalmente no foco do reestabelecimento das funções da floresta. No entanto, outros aspectos da dinâmica da floresta, incluindo serapilheira, sementes, propágulos, microrganismos, entre outros, devem ser considerados.

Ainda é premente a necessidade da realização de mais estudos que abordem os atributos do solo. Com esses estudos poderão ser estabelecidos padrões regionalizados para esse importante indicador, tornando seu uso corriqueiro em projetos técnicos, ou seja, tornando o solo um indicador aplicado e regionalizado de sustentabilidade dos ecossistemas florestais, tanto nos aspectos do manejo sustentável como nos da restauração de florestas degradadas.

\section{STATUS DA SUBMISSÃO}

Recebido: 29 abr., 2013

Aceito: 21 mar., 2015

\section{AUTOR(ES) PARA CORRESPONDÊNCIA}

\section{José Henrique Tertulino Rocha}

Escola Superior de Agricultura Luiz de Queiroz ESALQ, Universidade de São Paulo - USP,

CEP 13418-260, Piracicaba, SP, Brasil

e-mail: rocha.jht@gmail.com

\section{REFERENNCIAS}

Abreu SL, Reichert JM, Reinert DJ. Escarificação mecânica e biológica para a redução da compactação em argissolo franco-arenoso sob plantio direto. Revista Brasileira de Ciencia do Solo 2004; 28: 519-531.

Alleoni LRF, Camargo AO, Casagrande JC, Soares MR. Química dos solos altamente intemperizados. In: Melo VF, Alleoni LRF. Química e mineralogia do solo. Viçosa, MG: Sociedade Brasileira de Ciência do solo; 2009. vol. 2.

Alvares CA, Stape JL, Sentelhas PC, Gonçalves JLM, Sparovek G. Köppen's climate classification map for Brazil. Meteorologische Zeitschrift 2014; 22(6): 711-728. http:// dx.doi.org/10.1127/0941-2948/2013/0507.

Araújo R, Goedert WJ, Lacerda MPC. Qualidade de um solo sob diferentes usos e sob cerrado nativo. Revista Brasileira de Ciencia do Solo 2007; 31(5): 1099-1108. http://dx.doi.org/10.1590/S0100-06832007000500025.
Bayer C, Mielniczuk J. Características químicas do solo afetadas por métodos de preparo e sistemas de cultura. Revista Brasileira de Ciência do Solo 1997; 21: 105-112.

Beutler AN, Silva MLN, Curi N, Ferreira MM, Cruz JC, Pereira IA Fo. Resistência à penetração e permeabilidade de latossolo vermelho distrófico típico sob sistemas de manejo na região dos cerrados. Revista Brasileira de Ciencia do Solo 2001; 25(1): 167-177. http://dx.doi.org/10.1590/ S0100-06832001000100018.

Blake GR, Hartge KH. Bulk density. In: KLUTE A, editor. Methods of soil analysis. 2. ed. Madison: ASA/SSSA; 1986. Agronomy Monography n. 9.

Brady NC, Weil RR. The Nature and properties of soils. 13. ed. New Jersey: Prentice Hall Upper Saddle River; 2002.

Brun EJ. Matéria orgânica do solo em plantios de Pinus taeda e P. elliottii em duas regiões do Rio Grande Do Sul. Santa Maria - RS [tese]. Santa Maria, RS: Universidade Federal de Santa Maria; 2008. 119 p.

Castro C Fo, Muzilli O, Podanosch AL. Estabilidade dos agregados e sua relação com o teor de carbono orgânico num latossolo roxo distrófico, em função de sistemas de plantio, rotações de culturas e métodos de preparo das amostras. Revista Brasileira de Ciencia do Solo 1998; 22(3): 527-538. http://dx.doi.org/10.1590/S010006831998000300019.

Cerri CC, Volkoff B, Eduardo BP. Efeito do desmatamento sobre a biomassa microbiana em latossolo amarelo da Amazônia. Revista Brasileira de Ciência do Solo 1985; 9(1): 1-4.

D’Andréa AF, Silva MLN, Curi N, Siqueira JO, Carneiro MAC. Atributos biológicos indicadores da qualidade do solo em sistemas de manejo na região do cerrado no sul do estado de Goiás. Revista Brasileira de Ciencia do Solo 2002; 26(4): 913-923. http://dx.doi.org/10.1590/S010006832002000400008 .

Empresa Brasileira de Pesquisa Agropecuária - Embrapa. Centro Nacional de Pesquisa de Solos. Manual de métodos de análise de solo. 2. ed. Rio de Janeiro: Embrapa Solos; 1997. $212 \mathrm{p}$.

Empresa Brasileira de Pesquisa Agropecuária - Embrapa. Centro Nacional de Pesquisa de Solos. Sistema brasileiro de classificação de solos. 2. ed. Rio de Janeiro: Embrapa; 2006. 306 p.

Godinho TO, Caldeira MVW, Rocha JHT, Caliman JP, Vieira M. Fertilidade do solo e nutrientes na serapilheira em fragmento de Floresta Estacional Semidecidual. Revista Ecologia e Nutrição Florestal 2013; 1: 97-109. http://dx.doi. org/10.13086/2316-980x.v01n03a01.

Islam KR, Weil RR. Land use effects on soil quality in a tropical forest ecosystem of Bangladesh. Agricultural Ecosysistem Enviroment 2000; 79(1): 9-16. http://dx.doi. org/10.1016/S0167-8809(99)00145-0. 
Kiehl JE. Manual de edafologia. São Paulo: Ceres; 1979. $262 \mathrm{p}$.

Leite FP. Relações nutricionais e alterações de características químicas de solo da região do Vale do Rio Doce pelo cultivo do eucalipto [tese]. Viçosa: Universidade Federal de Viçosa; 2001. 72 p.

Lima AMN, Silva IR, Neves JCL, Novais RF, Barros NF, Mendonça ES et al. Frações da matéria orgânica do solo após três décadas de cultivo de eucalipto no Vale do Rio Doce-MG. Revista Brasileira de Ciencia do Solo 2008; 32(3): 1053-1063. http://dx.doi.org/10.1590/S010006832008000300014 .

Melloni R, Melloni EGP, Alvarenga MIN, Vieira FBM. Avaliação da qualidade de solos sob diferentes coberturas florestais e de pastagem no sul de Minas Gerais. Revista Brasileira de Ciencia do Solo 2008; 32(6): 2461-2470. http://dx.doi.org/10.1590/S0100-06832008000600023.

Melo ACG, Miranda DLC, Durigan G. Cobertura de copas como indicador de desenvolvimento estrutural de reflorestamentos de restauração de matas ciliares no médio vale do Paranapanema, SP, Brasil. Revista Árvore 2007; 31(2): 321-328. http://dx.doi.org/10.1590/S010067622007000200015

Meurer EJ. Fatores que influenciam o crescimento e desenvolvimento das plantas. In: NOVAES RF, ALVAREZ VH, BARROS NF, FONTES RLF, CANTARUTTI RB, NEVES JCL, editores. Fertilidade do solo. Viçosa: Sociedade Brasileira de Ciência do Solo; 2007. p. 375-470.

Moreira A, Malavolta E. Dinâmica da matéria orgânica e da biomassa microbiana em solo submetido a diferentes sistemas de manejo na Amazônia ocidental. Pesquisa Agropecuaria Brasileira 2004; 39(11): 1103-1100. http:// dx.doi.org/10.1590/S0100-204X2004001100008.

Oliveira GC, Dias MS Jr, Resck DVS, Curi N. Caracterização química e físico-hídrica de um latossolo vermelho após vinte anos de manejo e cultivo do solo. Revista Brasileira de Ciencia do Solo 2004; 28(2): 327-336. http://dx.doi. org/10.1590/S0100-06832004000200011.

Prezotti LC. Manual de recomendação de calagem $e$ adubação para o Estado do Espírito Santo. Vitória, ES: SEEA; 2007. 305 p.

Reichert JM, Reinert DJ, Braida JA. Qualidade dos solos e sustentabilidade de sistemas agrícolas. Ciência e Ambiente 2003; 27(2): 29-48.
Reinert DJ, Albuquerque JA, Reichert JM, Aita C, Andrada MMC. Limites críticos de densidade do solo para o crescimento de raízes de plantas de cobertura em argissolo vermelho. Revista Brasileira de Ciencia do Solo 2008; 32(5): 1805-1816. http://dx.doi.org/10.1590/ S0100-06832008000500002.

Rodrigues LA, Carvalho DA, Oliveira AT Fo, Curi N. Efeitos de solos e topografia sobre a distribuição de espécies arbóreas em um fragmento de floresta estacional semidecidual, em Luminárias, MG. Revista Árvore 2007; 31(1): 25-35. http://dx.doi.org/10.1590/S0100-67622007000100004.

SAS Institute Inc. - SAS. SAS/STAT 9.2 user's guide. Cary, NC: SAS Institute Inc; 2008.

Silveira AM, Victoria RL, Ballester MV, Camargo PB, Martinelli LA, Piccolo MC. Simulação dos efeitos das mudanças do uso da terra na dinâmica de carbono no solo na bacia do Rio Piracicaba. Pesquisa Agropecuária Brasileira 2000; 35(2): 389-399. http://dx.doi.org/10.1590/ S0100-204X2000000200018.

Souza ZM, Alves MC. Propriedades químicas de um latossolo vermelho distrófico de cerrado sob diferentes usos e manejos. Revista Brasileira de Ciencia do Solo 2003; 27(1): 133-139. http://dx.doi.org/10.1590/S010006832003000100014 .

Trumbore SE, Davidson EA, Barbosa de Camargo PB, Nepstad DC, Martinelli LA. Belowground cycling of carbon in forests and pastures of eastern Amazonia. Global Biogeochemical Cycles 1995; 9(4): 515-528. http://dx.doi. org/10.1029/95GB02148.

Van Raij B, Andrade JC, Cantarella H, Quaggio JA. Análise química para avaliação da fertilidade de solos tropicais. 4 . ed. Campinas: Fundação Cargill; 2001. 343 p.

Vital ART, Guerrini IA, Franken WK, Fonseca RCB. Produção de serapilheira e ciclagem de nutrientes de uma floresta estacional semidecidual em zona ripária. Revista Árvore 2004; 28(6): 793-800. http://dx.doi.org/10.1590/ S0100-67622004000600004.

Walkley A, Black IA. An examination of the Degtjareff method for determining soil organic matter and a proposed modification of the chromic acid titration method. Soil Science 1934; 37(1): 29-38. http://dx.doi. org/10.1097/00010694-193401000-00003. 